\title{
ALMOST LOCALLY CONNECTED SPACES
}

\author{
VINCENT J. MANCUSO
}

(Received 25 July 1980; revised 28 November 1980 and 7 January 1981)

Communicated by J. H. Rubinstein

\begin{abstract}
This paper introduces the concept of an almost locally connected space. Every locally connected space is almost locally connected, and the concepts are equivalent in the class of semi-regular spaces. Almost local connectedness is hereditary for regular open subspaces, is preserved by continuous open maps, but not generally by quotient maps. It is productive in the presence of almost-regularity.

1980 Mathematics subject classification (Amer. Math. Soc.): 54 D 05; 54 G 10; 54 D 10.

Keywords and phrases: Almost locally connected, locally connected, regular open, almost continuous, almost regular.
\end{abstract}

\section{Introduction}

This paper introduces the concept of an almost locally connected space. Every locally connected space is almost locally connected, and the concepts are equivalent in the semi-regular spaces of Stone (1937). Almost local connectedness is hereditary for regular open subspaces, is preserved by continuous open maps, but not generally by quotient maps. It is productive in the presence of almost regularity, Singal and Arya (1969).

\section{Preliminaries}

In this paper all spaces are $T_{1}$ and all maps are onto. A neighborhood of a point $p$ in a space $X$ will mean an open set of $X$ containing $p$. If $A \subseteq X$, the 
closure of $A$ in $X$ and the interior of $A$ in $X$ will be denoted by $\operatorname{cl}(A)$ and $\operatorname{int}(A)$ respectively. If $B \subseteq A \subseteq X$, the closure of $B$ and the interior of $B$ considered as a subspace of $A$ will be denoted by $\operatorname{cl}_{A}(B)$ and int $t_{A}(B)$ respectively.

Recall that an open set $G$ in $X$ is called regular open provided $G=\operatorname{int}(\operatorname{cl}(G))$. A regular open neighborhood of a point $p$ in $X$ will mean a regular open set containing $p$. A regular open cover $\mathcal{U}$ of a space $X$ is a cover of $X$ each member of which is a regular open set. A space $X$ is said to be semi-regular if $X$ has a basis consisting of regular open sets. This concept is originally due to Stone (1937). Regular spaces are semi-regular, but the converse is not true, Steen and Seebach (1970), Example 60.

A map $f: X \rightarrow Y$ is almost continuous provided $f^{-1}(G)$ is open in $X$ whenever $G$ is regular open in $Y$. A map $f: X \rightarrow Y$ is almost open provided $f(G)$ is open in $Y$ whenever $G$ is regular open in $X$, Singal and Singal (1968).

\section{Almost locally connected spaces}

Definition 3.1. A space $X$ is almost locally connected at $p \in X$ if given a regular open neighborhood $G$ of $p$, there is a connected neighborhood $V$ of $p$ such that $V \subseteq G . X$ is almost locally connected provided $X$ is almost locally connected at each of its points.

Henceforth, we shall abbreviate "almost locally connected" to a.l.c.

REMARK 3.2.(a) Since $X$ itself is a regular open set, it is clear that each point in an a.l.c. space is contained in a connected neighborhood.

(b) Clearly, every locally connected space is a.l.c., but Example 3.4 shows that the converse is not true even if the space is completely Hausdorff.

The reader can supply the easy proof of the following proposition.

Proposition 3.3. Let $X$ be semi-regular. $X$ is a.l.c. if and only if $X$ is locally connected.

EXAMPLE 3.4. Let $R$ be the set of reals and let $\tau$ be the topology on $R$ generated by the union of $\tau_{1}$, the usual topology on $R$, and $\tau_{2}$, the topology of countable complements on $R$. This is Example 63 in Steen and Seebach (1970). It is not difficult to see that $(R, \tau)$ is not locally connected at any irrational. However, the only regular open sets in $(R, \tau)$ are those which are regular open in $\tau_{1}$. Therefore, it follows that $(R, \tau)$ is a.l.c. 
THEOREM 3.5. The following statements are equivalent.

(1) $X$ is a.l.c.

(2) Components of regular open sets in $X$ are open in $X$.

(3) If $G$ is a neighborhood of $x \in X$, there is a connected neighborhood $V$ of $x$ such that $V \subseteq \operatorname{cl}(G)$.

PROof. The equivalence of (1) and (2) follows by a slight modification of the argument for locally connected spaces and open sets.

(1) implies (3): If $G$ is a neighborhood of $x$, int( $\operatorname{cl}(G))$ is a regular open neighborhood of $x$ and so there is a connected neighborhood $V$ of $x$ such that $V \subseteq \operatorname{int}(\operatorname{cl}(G)) \subseteq \operatorname{cl}(G)$.

(3) implies (1): If $G$ is a regular open neighborhood of $x$, there is a connected neighborhood $V$ of $x$ such that $V \subseteq \operatorname{cl}(G)$. Therefore, $V \subseteq \operatorname{int}(\operatorname{cl}(G))=G$ since $G$ is regular open.

REMARK 3.6.(a) We may assume the set $V$ in (3) of Theorem 3.5 is regular open or else replace it by int(cl(V)) which is connected if $V$ is connected.

(b) It is now obvious that a component in an a.l.c. space is open and so an a.l.c. totally disconnected space must be discrete. Of course, this is well known for locally connected spaces.

Unlike locally connected spaces, an open subspace of an a.l.c. space need not be a.l.c.

EXAMPLE 3.7. Consider the open subspace of irrationals in Example 3.4. Note, however, that this subspace is not regular open.

Theorem 3.8. Let $A$ be a regular open subspace of an a.l.c. space $X$. Then $A$ is a.l.c.

Proof. Let $p \in A$ and let $B$ be a regular open neighborhood of $p$ in $A$. We first establish that $B$ is a regular open neighborhood of $p$ in $X$.

Now

$$
\begin{aligned}
B & =\operatorname{int}_{A}\left(\mathrm{cl}_{A}(B)\right)=\operatorname{int}\left(\mathrm{cl}_{A}(B)\right)=\operatorname{int}(\operatorname{cl}(B) \cap A) \\
& =\operatorname{int}(\operatorname{cl}(B)) \cap \operatorname{int}(A)=\operatorname{int}(\operatorname{cl}(B)) \cap A=\operatorname{int}(\operatorname{cl}(B)) .
\end{aligned}
$$

Note that this last equality follows since $A$ is regular open in $X$ and so $\operatorname{int}(\operatorname{cl}(B)) \subseteq \operatorname{int}(\operatorname{cl}(A))=A$.

Since $X$ is a.l.c., there is a connected neighborhood $V$ of $p$ in $X$ such that $V \subseteq B$. Clearly, $V$ is a connected neighborhood of $p$ in $A$. Since $p$ was arbitrary, $A$ is a.l.c. 
THeOrem 3.9. Let $X$ be a.l.c. and $p \in X$. Then $X-\{p\}$ is a.l.c.

Proof. Let $x \in X-\{p\}$. We show that $X-\{p\}$ is a.l.c. at $x$. We will consider cases.

Case 1. There is a neighborhood $U$ of $x$ such that $p \notin \operatorname{int}(\operatorname{cl}(U))$. Then since int $(\mathrm{cl}(U))$ is regular open in $X$ and $X$ is a.l.c., there is a connected neighborhood $V$ of $x$ in $X$ such that $V \subseteq \operatorname{int}(\operatorname{cl}(U))$. Clearly, $V$ is a connected neighborhood of $x$ in $X-\{p\}$.

Case 2. For every neighborhood $U$ of $x, p \in \operatorname{int}(\operatorname{cl}(U))$. We will show that $X-\{p\}$ satisfies (3) of Theorem 3.5. Let $G$ be a neighborhood of $x$ in $X-\{p\}$. Since $X$ is $T_{1}, G$ is open in $X$, and so by Remark 3.6(a) there is a connected regular open neighborhood $V$ of $x$ in $X$ such that $V \subseteq \operatorname{cl}(G)$. Let $V_{1}=V-$ $\{p\}$. Then $x \in V_{1} \subseteq \operatorname{cl}(G)-\{p\}$ which is the closure of $G$ in $X-\{p\}$. So, if $V_{1}$ is connected we are done.

If $V_{1}$ is not connected, let $V_{1}=A \cup B$, where $A \cap B=\varnothing$ and $A$ and $B$ are non-empty and open in $V_{1}$ and hence in $X$. Say $x \in A$. Then by Case 2, $p \in \operatorname{int}(\operatorname{cl}(A))$. Since $V$ is regular open, it is easy to show that $V=\operatorname{int}(\operatorname{cl}(A)) \cup$ int $(\operatorname{cl}(B))$, and since $A$ and $B$ are disjoint and open in $X$, it follows that $\operatorname{int}(\operatorname{cl}(A)) \cap \operatorname{int}(\operatorname{cl}(B))=\varnothing$. But this contradicts the connectedness of $V$. This completes the proof.

COROllaRY 3.10. Let $X$ be a.l.c., locally compact, non-discrete space with a dispersion point. Then $X$ is uncountable.

Proof. Suppose $X$ is countable. Let $p$ be the dispersion point of $X$. By Theorem 3.9, $X-\{p\}$ is a.l.c. and so by Remark 3.6(b), $X-\{p\}$ must be discrete. But $X$ is not discrete, and so $p$ must be the only non-isolated point of $X$. Being countable and locally compact, $X$ is a hemi-compact $k$-space. Therefore, by Siewiec (1976), Theorem 1.2, $X$ must be one of three easily described examples. It is easy to check that none of these examples is a.l.c. This contradiction proves the corollary.

EXAMPLE 3.11. Let $Q$ be the rationals with the usual induced topology. Let $Q^{*}=Q \cup\{p\}$ be the one point compactification of $Q$. This is Example 35 in Steen and Sebach (1970). $Q^{*}$ is a countable locally compact, in fact compact, nondiscrete $T_{1}$ space with dispersion point $p$. This apparent contradiction to Corollary 3.10 is dispelled once we note that $Q^{*}$ is not a.l.c. If $Q^{*}$ were a.l.c., then by Theorem 3.9 and Remark 3.6(b), $Q$ would be discrete. 
Definition 3.12. A space $X$ is almost regular if for each $x \in X$ and each neighborhood $M$ of $x$, there is a regular open neighborhood $V$ of $x$ such that $\operatorname{cl}(V) \subseteq \operatorname{int}(\operatorname{cl}(M))$, Singal and Arya (1969).

REMARK 3.13. Every regular space is almost regular. But there exist almost regular spaces which aren't even semi-regular. Example 3.4 is such a space which is also a.l.c.

THEOREM 3.14. Let $X$ be almost regular. The following statements are equivalent.

(1) $X$ is a.l.c.

(2) Every regular open cover of $X$ has a regular open refinement consisting of connected sets.

(3) Every regular open cover of $X$ has an open refinement consisting of connected sets.

Proof. (1) implies (2). Let $\mathcal{Q}=\left\{U_{\alpha}\right\}$ be a regular open cover of $X$. Let $\mathcal{V}=\left\{V_{\beta}\right\}$ consist of all components of all the $U_{\alpha} \in \mathcal{U}$. By Theorem 3.5, $\mathfrak{V}$ consists of open sets. Let $\mathscr{W}=\left\{\operatorname{int}\left(\operatorname{cl}\left(V_{\beta}\right) ; V_{\beta} \in \mathfrak{V}\right\}\right.$. Now $\mathcal{V}$ clearly refines $\mathcal{Q}$ and since each member of $\mathcal{U}$ is a regular open st, $\mathscr{W}$ also refines $\mathscr{U}$. Since $V_{\beta} \subseteq \operatorname{int}\left(\operatorname{cl}\left(V_{\beta}\right) \subseteq \operatorname{cl}\left(V_{\beta}\right)\right.$ for each $\beta$, it is clear that each member of $\mathscr{W}$ is connected. Being the interior of a closed set, each member of $\mathscr{W}$ is a regular open set.

(2) implies (3). This is obvious.

(3) implies (1). Let $p \in X$ and let $G$ be a regular open neighborhood of $p$. Since $X$ is almost regular, there is by Theorem 2.2(b) of Singal and Arya (1969), a regular open neighborhood $U$ of $p$ such that $\operatorname{cl}(U) \subseteq G$. Now the two sets $\{G, X-\operatorname{cl}(U)\}$ is a regular open cover of $X$. Note that $X-\operatorname{cl}(U)$ is regular open since $U$ is. By (3) this cover has an open refinement $\mathcal{K}=\left\{H_{\beta}\right\}$ such that each $H_{\beta}$ is connected. Let $H_{\beta}(p)$ be that member of $\mathcal{K}$ such that $p \in H_{\beta}(p) \subseteq$ $G$. This proves that $X$ is a.l.c.

Defintion 3.15. A map $f: X \rightarrow Y$ is connected if $f(C)$ is connected in $Y$ whenever $C$ is connected in $X$, Pervin and Levine (1958).

THEOREM 3.16. Let $f: X \rightarrow Y$ be an almost open, almost continuous connected map. If $X$ is a.l.c. and $Y$ is almost regular, then $Y$ is a.l.c.

Proof. Since $Y$ is almost regular, it suffices to show that $Y$ satisfies condition (3) of Theorem 3.14. Let $\mathscr{U}=\left\{U_{\alpha}\right\}$ be a regular open cover of $Y$. Since $f$ is 
almost continuous, $f^{-1}(\mathcal{U})=\left\{f^{-1}\left(U_{\alpha}\right) ; U_{\alpha} \in \mathcal{U}\right\}$ is an open cover of $X$. Hence $\mathscr{W}=\left\{\operatorname{int}\left(\operatorname{cl}\left(f^{-1}\left(U_{\alpha}\right)\right)\right) ; U_{\alpha} \in \mathcal{Q}\right\}$ is a regular open cover of $X$. Using the argument of (1) implies (2) in Theorem 3.14, we can find a regular open refinement $\mathcal{H}=\left\{\boldsymbol{H}_{\beta}\right\}$ of $\mathscr{W}$ such that each member of $\mathcal{K}$ is connected. Since $f$ is almost open and connected, each $f\left(H_{\beta}\right)$ is connected and open in $Y$. We need only show that $f(\mathcal{K})=\left\{f\left(H_{\beta}\right) ; H_{\beta} \in \mathcal{K}\right\}$ is a refinement of $\mathcal{U}$. Since $\mathcal{K}$ covers $H$, it is clear that $f(H)$ covers $Y$. Let $H_{\beta} \in \mathcal{K}$. Since $\mathcal{H}$ refines $\mathcal{W}$, there is an $\alpha$ such that $H_{\beta} \subseteq \operatorname{int}\left(\operatorname{cl}\left(f^{-1}\left(U_{\alpha}\right)\right)\right) \subseteq \operatorname{cl}\left(f^{-1}\left(U_{\alpha}\right)\right)$. By Long and Carnahan (1973) Theorem 6, it follows that $f\left(\operatorname{cl}\left(f^{-1}\left(U_{\alpha}\right)\right)\right) \subseteq \operatorname{cl}\left(U_{\alpha}\right)$. Therefore, $f\left(H_{\beta}\right) \subseteq \operatorname{cl}\left(U_{\alpha}\right)$. But since $f\left(H_{\beta}\right)$ is open, $f\left(H_{\beta}\right) \subseteq \operatorname{int}\left(\operatorname{cl}\left(U_{\alpha}\right)\right)$. Since $U_{\alpha}$ is regular open, this latter set is just $U_{\alpha}$. This completes the proof.

LEMMA 3.17. Let $f: X \rightarrow Y$ be an open almost continuous map. If $G$ is regular open in $Y$, then $f^{-1}(G)$ is regular open in $X$.

Proof. Let $G$ be regular open in $Y$. By the Corollary after Theorem 7 in Long and Carnahan (1973), $\operatorname{cl}\left(f^{-1}(G)\right)=f^{-1}(\operatorname{cl}(G))$. Hence $\operatorname{int}\left(\operatorname{cl}\left(f^{-1}(G)\right)\right)=$ $\operatorname{int}\left(f^{-1}(\mathrm{cl}(G))\right)$. Since if is an open map, $\operatorname{int}\left(f^{-1}(\operatorname{cl}(G))\right) \subseteq f^{-1}(\operatorname{int}(\operatorname{cl}(G)))$. Since $G$ is regular open, this latter set is just $f^{-1}(G)$. Hence int $\left(\operatorname{cl}\left(f^{-1}(G)\right)\right) \subseteq f^{-1}(G)$. The reverse inclusion is obvious since $f^{-1}(G)$ is open in $X$. This proves that $f^{-1}(G)$ is regular open in $X$.

THEOREM 3.18. Let $f: X \rightarrow Y$ be an open almost continuous connected map. If $X$ is a.l.c., so is $Y$.

Proof. Let $G$ be a regular open neighborhood of $y \in Y$. By Lemma 3.17, $f^{-1}(G)$ is regular open in $X$. Since $X$ is a.l.c., there is for each $x \in f^{-1}(y)$ a connected neighborhood $N(x)$ of $x$ such that $N(x) \subseteq f^{-1}(G)$. Let $N=$ $\cup\left\{N(x) ; x \in f^{-1}(y)\right\}$. Since $f$ is a connected open map and $y \in f(N(x))$ for each $x \in f^{-1}(y)$, it is clear that $f(N)$ is a connected neighborhood of $y$ in $Y$. Moreover, $f(N) \subseteq G$. This proves that $Y$ is a.l.c.

Since an open continuous map is almost open, almost continuous and connected, the following corollary follows from Theorm 3.18.

Corollary 3.19. Let $f: X \rightarrow Y$ be an open continuous map. If $X$ is a.l.c. so is $Y$.

Unlike local connectedness, a.l.c. is not preserved by continuous closed maps and so not by quotient maps. 
Example 3.20. Consider the space $(R, \tau)$ of Example 3.4. Let $R / Q$ denote the quotient space obtained from $(R, \tau)$ by identifying the set $Q$ of rationals to a point. Since $Q$ is closed in $(R, \tau)$, the space $R / Q$ is $T_{1}$ and he quotient map $p$ : $(R, \tau) \rightarrow R / Q$ is a closed map.

We contend that $R / Q$ is not a.l.c. We will use brackets to denote a point in $R / Q$ and $[Q]$ will denote the "big" point in $R / Q$. Let $z$ be any irrational. It suffices to show that $R / Q$ is not a.l.c. at $[z]$. Let $a$ and $b$ be any rationals such that $a<z<b$ and let $G=\{[x] ; x \in(a, b), x$ irrational $\}$. It is not difficult to see that $G$ is a regular open neighborhood of $[z]$ in $R / Q$ such that $[Q] \notin G$. Now if $R / Q$ were a.l.c., there would exist a connected neighborhood $W$ of $[z]$ in $R / Q$ such that $W \subseteq G$. Let $h=p \mid R-Q$, the restriction to $p$ to $R-Q$. Then $h$ is a homeomorphism of $R-Q$ onto $R / Q-[Q]$. This is an exercise in Dugundji (1966), p. 125. Therefore, $h^{-1}(W)$ would be a connected open subset of irrationals in $(R, \tau)$. This is not possible since $\tau_{1} \subseteq \tau$.

We have the following product theorem for a.l.c. spaces.

THEOREM 3.21. Let $\left\{X_{\alpha} ; \alpha \in \mathbb{Q}\right\}$ be a family of spaces. Then $\Pi\left\{X_{\alpha} ; \alpha \in \mathbb{Q}\right\}$ is a.l.c. if and only if each $X_{\alpha}$ is a.l.c. and all but at most finitely many $X_{\alpha}$ are connected.

Proof. Suppose $\operatorname{II}\left\{X_{\alpha} ; \alpha \in \mathbb{Q}\right\}$ is a.l.c. Since projection maps are continuous and open maps, each $X_{\alpha}$ is a.l.c. by Corollary 3.19. By Remark 3.2(a), all but at most finitely many $X_{\alpha}$ are connected.

Conversely, let $G$ be a neighborhood of $x$ in $\Pi\left\{X_{\alpha} ; \alpha \in \mathbb{Q}\right\}$. Using the notation in Dugundji (1966), p. 98, we will let $U=\left\langle U_{\alpha_{1}}, \ldots, U_{\alpha_{k}}\right\rangle$ denote a basic open set in $\Pi\left\{X_{\alpha} ; \alpha \in \mathbb{Q}\right\}$ such that $x \in U \subseteq G$. Since each $X$ is a.l.c., there exist $V_{\alpha_{1}}, \ldots, V_{\alpha_{k}}$ connected and open in $X_{\alpha_{1}}, \ldots, X_{\alpha_{k}}$ respectively such that $x_{\alpha_{i}} \in V_{\alpha_{i}}$ and $V_{\alpha_{i}} \subseteq \operatorname{cl}\left(U_{\alpha_{i}}\right)$ for $i=1, \ldots, k$.

Let $\mathscr{B}$ be the finite subset of $\mathscr{Q}$ such that if $\beta_{j} \in \mathscr{B}$, then $X_{\beta}$ is not connected. For each $\beta_{j} \in \mathscr{B}$, let $V_{\beta_{j}}$ be a connected neighborhood of $x_{\beta_{j}}$, in $X_{\beta_{j}}$ (Remark 3.2(a)). Let $V=\left\langle V_{\alpha_{1}}, \ldots, V_{\alpha_{k}}, V_{\beta_{1}}, \ldots, V_{\beta_{n}}\right\rangle$. Clearly, $V$ is a connected neighborhood of $x$ in $\Pi\left\{X_{\alpha} ; \alpha \in \mathbb{Q}\right\}$. Moreover, it is easy to check that $V \subseteq \operatorname{cl}(G)$. By Theorem 3.5, we are done.

REMARK 3.22. The space $(R, \tau)$ in Example 3.20 is not compact. This raises the following questions. If $f: X \rightarrow Y$ is continuous with $X$ compact (and $T_{1}$ ) and a.l.c. is $Y$ a.l.c.? Or, is the property a.l.c. preserved by perfect maps? Note that the closed map $p$ in Example 3.20 is not perfect since $p^{-1}([Q])$ is not compact in $(R, \tau)$. 
Finally, the author would like to thank the referee for some helpful comments. In the original version of this paper, Theorem 3.9 was proved for $T_{2}$ spaces and we asked if this theorem held for $T_{1}$ spaces. The referee answered this question affirmatively and supplied the proof of Case 2. He also pointed out the equivalence of (1) and (3) of Theorem 3.5. This allowed the author to remove the hypothesis that each $X$ be almost regular in the original version of the product Theorem 3.21.

\section{References}

J. Dugundji (1966), Topology (Allyn and Bacon, Boston).

P. E. Long and D. A. Carnahan (1973), 'Comparing almost continuous functions', Proc. Amer. Math. Soc. 38, 413-418.

W. J. Pervin and N. Levine (1958), 'Connected mappings of Hausdorff spaces', Proc. Amer. Math. Soc. 9, 488-496.

M. K. Singal and S. P. Arya (1969), 'On almost regular spaces', Glasnik Mat. 4, 89-99.

M. K. Singal and A. R. Singal (1968), 'Almost-continuous mappings', Yokohama Math. J. 16, 63-73.

F. Siwiec (1976), 'Countable spaces having exactly one nonisolated point $I$ ', Proc. Amer. Math. Soc. 57, 345-348.

L. A. Steen and J. A. Seebach Jr. (1970), Counterexamples in topology (Holt, Rinehart and Winston, New York).

M. H. Stone (1937), 'Applications of the theory of Boolean rings to general topology', Trans. Amer. Math. Soc. 41, 375-481.

Department of Mathematics

St. John's University

Jamaica, New York 11439

U.S.A. 